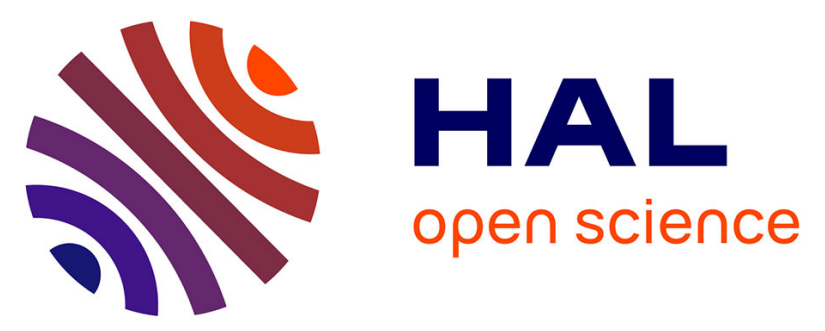

\title{
New Hybrid FE-FV Method for Computing Current Distribution in 2-D Superconductors: Application to an HTS Cylinder in Transverse Magnetic Field
} Abelin Kameni, Denis Netter, Frédéric Sirois, Bruno Douine, Jean Lévêque

\section{- To cite this version:}

Abelin Kameni, Denis Netter, Frédéric Sirois, Bruno Douine, Jean Lévêque. New Hybrid FE-FV Method for Computing Current Distribution in 2-D Superconductors: Application to an HTS Cylinder in Transverse Magnetic Field. IEEE Transactions on Applied Superconductivity, 2009, 19 (3), pp. 2423 - 2427. 10.1109/TASC.2009.2018047 . hal-01236835

\section{HAL Id: hal-01236835 https://hal.science/hal-01236835}

Submitted on 2 Dec 2015

HAL is a multi-disciplinary open access archive for the deposit and dissemination of scientific research documents, whether they are published or not. The documents may come from teaching and research institutions in France or abroad, or from public or private research centers.
L'archive ouverte pluridisciplinaire HAL, est destinée au dépôt et à la diffusion de documents scientifiques de niveau recherche, publiés ou non, émanant des établissements d'enseignement et de recherche français ou étrangers, des laboratoires publics ou privés. 


\title{
New Hybrid FE-FV Method for Computing Current Distribution in 2-D Superconductors: Application to an HTS Cylinder in Transverse Magnetic Field
}

\author{
Abelin Kameni, Denis Netter, Frédéric Sirois, Senior Member, IEEE, Bruno Douine, Jean Lévêque
}

\begin{abstract}
This paper presents a new numerical method based on finite elements - finite volumes (FE-FV) for solving 2-D diffusion problems in high temperature superconductors (HTS). The approach does not involve directly the resistivity term $(\rho)$, generally used to model the $E(J)$ characteristic as a power law, i.e $E(J)=\rho(J) J$, with $\rho(J) \propto J^{n-1}$. Instead, we use a $J(E)$ constitutive law $J \propto E^{\frac{1}{n}}$, with $\vec{E}=E \vec{e}_{z}$ (a single component), which leads to a scalar non-linear differential equation. After presenting in details the developments, the method is tested in the case of a superconducting cylinder submitted to a transverse magnetic field. The current density obtained is compared to another numerical technique (the semi-analytical method) in order to validate the results. Although not fully optimized yet, it appears that the proposed method is very stable, especially for large n-values (greater than 100).
\end{abstract}

Index Terms-Diffusion processes, electromagnetic analysis, finite-element methods (FEMs), high-temperature superconductors, integral equations, numerical analysis.

\section{INTRODUCTION}

$\mathbf{T}$ HE numerical models characterizing superconducting materials generally intend to solve Maxwell's equations, complemented by the non-linear constitutive equation $E(J)=$ $E_{c}\left(J / J_{c}\right)^{n}$ (power law characteristic) [1]. The use of a resistivity term $\rho$, such as $E=\rho(J) J$, is the most common approach to treat the non-linearity. The resulting equations are then "semilinear", and their resolutions depends on the calculation of $\rho(J)$. For instance, this is the underlying approach used by commercial finite element packages such as COMSOL Multiphysics or Flux2D. However, in the case of large $n$ values, this approach is prone to numerical oscillations and convergence problems. Others schemes using integral formulas in a semi-analytical form were proposed as an alternative to finite elements, but the same limitations occur for large $n$ values [2], [3].

In this paper, we present a model combining Maxwell's equations directly to a $J(E)$ power law $J(E)=J_{c}\left(E / E_{c}\right)^{\frac{1}{n}}$. We assume the low frequency regime, as well as linear permittivity and permeability, such that $\nabla \cdot \vec{E}=0$ and $\vec{B}=\mu_{0} \vec{H}$. Under these assumptions, the electric field is the solution of a non-linear parabolic diffusion equation:

$$
\nabla^{2} u=c \frac{\partial \beta(u)}{\partial t}
$$

Manuscript received August 17, 2008; revised December 19, 2008. This work was supported by XXXXXXXXXXXXXXXX.

A. Kameni, D. Netter, B. Douine and J. Lévêque are with Groupe de Recherche en Electronique et Electrotechnique de Nancy, France (e-mails: abelin.kameni@green.uhp-nancy.fr, denis.netter@green.uhp-nancy.fr, bruno.douine@green.uhp-nancy.fr, jean.leveque@green.uhp-nancy.fr).

F. Sirois is with École Polytechnique de Montréal, Montréal, QC H3C 3A7, Canada (e-mail: f.sirois@polymtl.ca) with, $u=E / E_{c}, \beta(u)=u^{\alpha}=J / J_{c}, \alpha=1 / n$ and $c=$ $\mu_{0} J_{c} / E_{c}$.

This type of equation is often encountered in fluid mechanics and porous media. The numerical recipes developed in applied mathematics for these non-linear parabolic equations have been the subject of many works in the past. Among them, the finite volume (FV) method have been proposed and thoroughly justified and validated [4].

When applied to our case, there is no need to consider explicitly a resistivity term, and the discrete problem can remain fully nonlinear. The fixed point theorem proves the existence of a discrete solution. The unknowns would correspond to the mean value of $u$ (the normalized electric field) on each cell of the finite volume mesh. Nevertheless, it remains difficult to obtain the gradient of a mean value on a finite volume mesh, although this is required since $\nabla^{2} u=\nabla \cdot(\nabla u)$. The discretization of the convective term (i.e. the divergence) containing a gradient therefore requires a specific treatment [5].

Footbridges between finite volumes (FV) and finite elements (FE) have been developed for convection-diffusion problems. They are used to connect the solutions FE and FV discretizations [6]. In the case of dual meshing, where the cells of finite volume mesh are constructed around the triangular mesh, it is possible to replace the FV discrete form of the convective term by a FE discrete form of the diffusive term (i.e. the Laplacian). These mixed schemes combine the advantages of both methods and are known to be robust, stable and efficient in the case of non-linear parabolic equations [7].

\section{Modeling}

\section{A. The differential problem}

The development presented below is valid for a single 2-D conducting domain (infinite length along $z$ axis). No transport current is considered for now, but an uniform applied field can be applied along an arbitrary axis in the $x-y$ plane. We also assume parameters $E_{c}, J_{c}$ and $n$ as being constants. When the external magnetic field depends on time according to $\vec{B}(t)=$ $B_{x}(t) \overrightarrow{e_{x}}+B_{y}(t) \overrightarrow{e_{y}}$, the induced electric field $\vec{E}=E(x, y, t) \overrightarrow{e_{z}}$ in the superconductor is the solution of the non-linear diffusion equation (1).

The Faraday's law and the continuity of the magnetic field lead to a boundary condition on $\nabla u$ :

$$
\nabla \times \vec{E}=\mathcal{A} \nabla E(x, y, t)=-\frac{\partial \vec{B}}{\partial t},
$$




$$
E_{c} \nabla u \cdot \vec{\nu}=-\mathcal{A}^{-1} \frac{\partial \vec{B}}{\partial t} \cdot \vec{\nu}
$$

where $\nu$ is the exterior normal to the domain boundary, and $\mathcal{A}=$ $\left(\begin{array}{cc}0 & 1 \\ -1 & 0\end{array}\right)$.

Our goal is therefore to solve the following differential system in accordance with the discretization rules presented in the next section :

$$
(S)\left\{\begin{array}{l}
c \frac{\partial \beta(u)}{\partial t}-\nabla^{2} u=0 \\
\nabla u \cdot \vec{\nu}=C_{b}(t) \\
u(t=0)=0
\end{array}\right.
$$

with $C_{b}(t)=-E_{c}^{-1} \mathcal{A}^{-1} \frac{\partial \vec{B}}{\partial t} \cdot \vec{\nu}$

\section{B. Mathematical formalism and weak formulation}

The problem domain is the space-time space $Q_{T}=\Omega \times[0, T]$. The FE and FV discretization methods consist in solving a weak formulation of the differential system (4), which is expressed as an integral equation, written in the appropriate spaces:

* The Hilbert space on $\Omega, L^{2}(\Omega)$ and the scalar product $(u, v)_{L^{2}}=\int_{\Omega} u v d \Omega$.

* The sub-space $H^{1}(\Omega)=\left\{u \in L^{2}(\Omega), \nabla u \in L^{2}(\Omega)\right\}$

* The space $L^{\infty}\left(0, T ; L^{2}(\Omega)\right): \beta(u)$ is bounded on $Q_{T}$ and at any given time $t, \beta(u) \in L^{2}(\Omega)$

The weak formulation is the projection on a distribution space of the differential system, when the later is approximated by a set of basis functions $\varphi_{i}$. The final equation (weak form) of our problem is obtained by multiplying the diffusion equation (4) by the test function $\varphi$ and integrating it on $Q_{T}$, i.e.

$$
\int_{0}^{T} \int_{\Omega} c \frac{\partial \beta(u)}{\partial t} \varphi d \Omega d t-\int_{0}^{T} \int_{\Omega} \nabla^{2} u \varphi d \Omega d t=0
$$

\section{DisCRETIZATION}

\section{A. Temporal discretization}

We chose to work with a prescribed partitioning of the time interval $[0, T]$ in $p+1$ points, which results in a series of discrete times $\left(t_{p}\right)_{0 \leq p \leq P}$. The time step is defined by $\Delta t_{p}=t_{p+1}-t_{p}$, and is small enough to use:

* The rectangular method for the integration on the interval $\left[t_{p}, t_{p+1}\right]$

$\star$ Finite differences for the calculation of the derivatives.

Let's use the notation $u\left(x, y, t_{p}\right)=u^{p}$ for describing the solution at time $t_{p}$. On each interval $\left[t_{p}, t_{p+1}\right]$, we have the following integral equation on $\Omega$ :

$$
c \int_{\Omega} \frac{\beta\left(u^{p+1}\right)-\beta\left(u^{p}\right)}{\triangle t_{p}} \varphi d \Omega-\int_{\Omega} \nabla^{2} u^{p+1} \varphi d \Omega=0 .
$$

The discrete problem is obtained by projecting this integral equation on the basis functions $\varphi_{i}$, defined on a finite dimension approximation subspace. These subspaces are built from the decomposition of the domain in triangles (FE) or control volumes $(\mathrm{FV})$, as shown in Fig. 1.

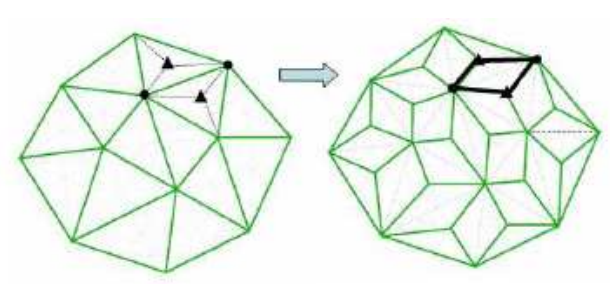

Fig. 1: FE triangular mesh (left) and FV control volumes (right): to build a FV cell from the FE mesh, we link the centers of gravity of two contiguous triangles through the vertex of their common edge.

\section{B. Finite element discretization}

For a set of nodes defining the triangular mesh, we denote $\mathcal{X}$ the approximation space determined by the basis functions $\varphi_{i}$, chosen as piecewise linear. Finding a FE discrete problem for (6) depends on the existence of the projection of $\beta(u)$ on $\mathcal{X}$. In our case, the existence is impossible because $\beta^{\prime}(0) \hookrightarrow+\infty$. This difficulty is avoided in Finite volume discretization.

But, the discretization of the Laplace operator is easy and wellknown [8]. It involves a diffusion matrix $S$, whose elements $S_{i, j}$ are the diffusion coefficients between the nodes $N_{i}$ and $N_{j}$, corresponding to the unknowns $u_{i}$ and $u_{j}$. For $u=\sum_{j} u_{j} \varphi_{j}$,

$$
\int_{\Omega} \nabla^{2} u \varphi_{i} d \Omega=\underbrace{-\int_{\Omega} \nabla u \cdot \nabla \varphi_{i} d \Omega}_{\text {Diffusion }}+\underbrace{\int_{\Gamma} \nabla u \cdot \varphi_{i} \vec{\nu} d \Gamma}_{\text {Boundary }}
$$

The diffusive term $\int_{\Omega} \nabla u \cdot \nabla \varphi_{i} d \Omega=\sum_{j} S_{i, j} u_{j}$ is a function of $S_{i, j}=\sum_{K \in \mathcal{I}_{h}} \int_{K} \nabla \varphi_{i} \cdot \nabla \varphi_{j} d K$

\section{Finite volume discretization}

The basis functions of the FV approximation space, denoted $\mathcal{V}$, are the indicatrix functions $1_{D}$ of the cells $D$ of the FV mesh $\left(1_{D}(x)=1\right.$ if $x \in D$, and $1_{D}(x)=0$ elsewhere $)$. The unknowns are the mean value of the solution (normalized electric field) on each cell. The definition of mean value is assumed by the continuity property of the function. Remembering that $\beta(u)$ is a non-linear but otherwise continuous function, the non-linearity is easily treated with the FV approach and the first integral in (6) is proportionnal of the mean value of $\beta$ on $D$.

Let's define $\beta_{D}^{p}=\left\langle\beta\left(u^{p}\right)\right\rangle_{D}$ be the mean values of $\beta(u)$ on cell $D$ at time $t=t_{p}$. We obtain:

$$
\int_{D} \frac{\beta\left(u^{p+1}\right)-\beta\left(u^{p}\right)}{\triangle t_{p}} 1_{D} d D=\frac{\beta_{D}^{p+1}-\beta_{D}^{p}}{\triangle t_{p}}|D|,
$$

where $|D|$ is the area of the FV element.

The FV method is based on the strict imposition of the flux conservation through the boundaries of each contiguous FV cells. In practice, it uses the divergence theorem to evaluate the convection through boundary integrals. The total outward flux $\Phi$ from a given cell $D$ and time step $p$ is given by:

$$
\Phi=\int_{D} \nabla \cdot\left(\nabla u^{p}\right) d D=\int_{\partial D} \nabla u^{p} \cdot \vec{v} d(\partial D),
$$


where $\vec{v}$ is the outward-pointing normal vector.

In terms of the discrete problem, the total outward flux from cell $D_{i}$ is the sum of the fluxes riding through each edge of the cell. The flux between two contiguous cells $D_{i}$ and $D_{j}$ therefore corresponds to $\Phi_{i, j}$. The FV formulation depends on the choice of the flux functions $\Phi_{i, j}$, which are chosen such that $\Phi_{i, j}=-\Phi_{j, i}$. The discrete equation on each cell can therefore be written as:

$$
\int_{\partial D} \nabla u^{p} \cdot \vec{v} d(\partial D)=\sum_{D_{j} \in \mathcal{N}\left(D_{i}\right)}\left|D_{i} \cap D_{j}\right| \Phi_{i, j}\left(u_{i}, u_{j}\right),
$$

where $\mathcal{N}\left(D_{i}\right)$ is the set of the contiguous cells of $D_{i}$. Unfortunately, approximating the gradient of the mean value is difficult. To solve this problem, we use footbridges between the FE and FV approximation spaces. These footbridges will allow expressing the fluxes $\Phi_{i, j}$ as a function of the $S_{i, j}$ terms.

\section{The mixed scheme}

When dual meshing is used to generate the FV cells, the footbridges beetween the FE and the FV approximation spaces are well described in [6]. Let $u^{F E}$ and $u^{F V}$ be the respective solutions of the FE and FV approaches. The footbridges operators $\pi_{E V}$ and $\pi_{V E}$ are projections defined as:

$\star \mathrm{FV}(\mathcal{V}) \rightarrow \mathrm{FE}(\mathcal{X}) \pi_{E V}\left(u^{F V}\right)=u^{F E}$ is the solution of the FE discretization and satisfies:

$$
\int_{\Omega}\left|\pi_{E V} u-u\right|^{2} d \Omega=\min _{w \in \mathcal{X}} \int_{\Omega}|w-u|^{2} d \Omega
$$

$\star \mathrm{FE}(\mathcal{X}) \rightarrow \mathrm{FV}(\mathcal{V}) \pi_{V E}\left(u^{F E}\right)=u^{F V}$ is the solution of the

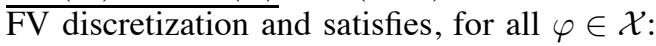

$$
\int_{\Omega} \pi_{V E} u \varphi d \Omega=\int_{\Omega} u \varphi d \Omega
$$

These footbridges can be used to show that mixing the two discretizations (FE and FV) is possible only if $u^{F E}=u^{F V}$. The existence of the footbridge operators $\pi_{E V}$ and $\pi_{V E}$ defining in (11) and (12) ensures the required equality and have been proved in previous papers, e.g. [6].

For the kind of dual meshing considered here, each cell $D_{i}$ of the FV mesh can be associated with one node $N_{i}$ of the FE mesh, as shown in Fig. 2.

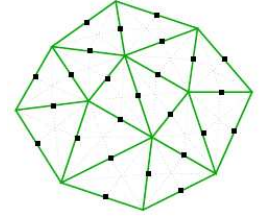

(a) The nodes $N_{i}$ correspond to the middle of the edges

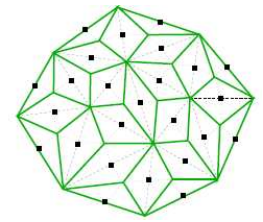

(b) The nodes $N_{i}$ corresponds to the center of the cells $D_{i}$
Fig. 2: Association of each node of the FE mesh to the cells of the FV mesh.

As a result, it is possible to calculate $\Phi_{i, j}$ as a function of $S_{i, j}$. On the internal FV cells (cells with 4 edges), we can consider that the FV convection and the FE diffusion are equal, i.e.

$$
\int_{D} \nabla \cdot(\nabla u) d D=\int_{\Omega} \nabla u \cdot \nabla \varphi_{i} d \Omega
$$

$$
\sum_{D_{j} \in \mathcal{N}\left(D_{i}\right)}\left|D_{i} \cap D_{j}\right| \Phi_{i, j}\left(u_{i}, u_{j}\right)=-\sum_{j} S_{i, j} u_{j}
$$

It is important that these expressions keep the local conservation property of the FV formulation $\left(\Phi_{i, j}=-\Phi_{j, i}\right)$. This is the case if we define $S_{i, i}=-\sum_{j \neq i} S_{i, j}$, where $S_{i, j}$ are positive coefficients. Under these conditions, we obtain:

$$
\left|D_{i} \cap D_{j}\right| \Phi_{i, j}\left(u_{i}, u_{j}\right)=S_{i, j}\left(u_{j}-u_{i}\right)
$$

The discrete problem (hybrid FE and FV numerical schemes) consists in calculating, at each time step $p+1$, a set of unknowns defined by $\left(u_{i}^{p+1}\right)_{1 \leq i \leq N}, N$ being the number of nodes in the FE mesh:

* For the internal cells (4 edges), one has:

$$
c \frac{\beta\left(u_{i}^{p+1}\right)-\beta\left(u_{i}^{p}\right)}{\triangle t_{p}}\left|D_{i}\right|-\sum_{j} S_{i, j} u_{j}^{p+1}=0
$$

* For the boundary cells (one edge belongs to the boundary $\Gamma$ ) and considering the boundary condition

$$
\int_{\Gamma} \vec{\nabla} u^{p+1} \cdot \varphi_{i} \vec{\nu} d \Gamma=\int_{\Gamma_{i}} C_{b}^{p+1} \varphi_{i} d \Gamma=\left|\Gamma_{i}\right| C_{b}^{p+1}
$$

one has:

$$
c \frac{\beta\left(u_{i}^{p+1}\right)-\beta\left(u_{i}^{p}\right)}{\triangle t_{p}}\left|D_{i}\right|-\sum_{j} S_{i, j} u_{j}^{p+1}-\left|\Gamma_{i}\right| C_{b}^{p+1}=0
$$

The existence of the discrete solution is proved by the fixedpoint theorem. The numerical computation uses the unknowns $v_{i}^{p+1}=\beta\left(u_{i}^{p+1}\right)$. Since $\beta^{-1}$ is continuous and differentiable, we can use a Newton-Raphson algorithm, i.e.

$$
u_{i}^{p, k}=\beta^{-1}\left(v_{i}^{p, k-1}\right)+\left.\left(\beta^{-1}\right)^{\prime}\right|_{v_{i}^{p, k-1}}\left(v_{i}^{p, k}-v_{i}^{p, k-1}\right)
$$

The convergence of this numerical scheme for each time step is obtained when the relative difference between two successive iterations $\epsilon=\sum_{i}\left[\frac{\left(v_{i}^{p, k}-v_{i}^{p, k-1}\right)}{v_{i}^{p, k}}\right]^{2}<10^{-9}$.

\section{NUMERICAL APPLICATION: LONG CYLINDER IN A TRANSVERSE MAGNETIC FIELD}

Let's consider a cylinder whose parameters are $R=10 \mathrm{~mm}$ (radius), $J_{c}=10^{8} \mathrm{~A} / \mathrm{cm}^{2}, E_{c}=10^{-4} \mathrm{~V} / \mathrm{m}^{-1}$ and $n=15$, and immersed in a transverse external field defined as

$$
\vec{B}=B_{\max } \sin (2 \pi f t) \vec{e}_{x}
$$

In the cases presented below, $f=50 \mathrm{~Hz}$, and we define $T_{0}=$ $1 / f=20 \mathrm{~ms}$. Based on the Bean model, the field required for full penetration is $B_{p}=2 \mu_{0} J_{c} R / \pi=0.8 T$. Setting $B_{\max }=B_{p}$ implies that the full penetration will occur at $t=T_{0} / 4$, where the applied field reaches its maximum value [9]. In the case of the power law characteristic with $n=15$, however, the full penetration is not obtained, as will be shown below.

In Fig. 3, we present a comparative result between numerical solutions of the normalized current density $\left(J / J_{c}\right)$ obtained with our hybrid method (HM) and with the semi-analytical method 
(SAM, [3]) at time $t=T_{0} / 4$. As can be seen, we find exactly the same solutions, to within numerical errors and mesh differences. As the SAM method was itself well validated against more classical finite element formulations, this proves the the formulation proposed is correct. Fig. 3 also reveals, as expected, that the full penetration is not reached because of the presence of the power law characteristic.

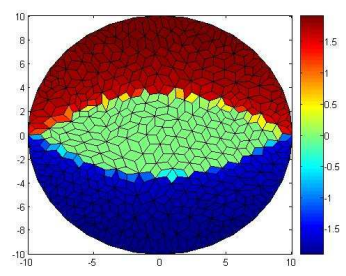

(a) Hybrid FEM-FVM

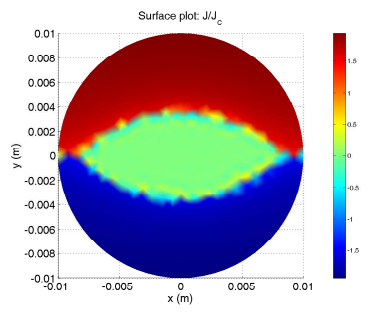

(b) SAM
Fig. 3: Comparison for $n=15$, at $t=T_{0} / 4$

As a further validation, we compared the results of both methods for $n=100$, as shown in Fig. 4 (for $t=T_{0} / 8$ ) and Fig. 5 (for $t=T_{0} / 4$ ). In this case, however, we observe different penetration rates, which is surprising a priori, given the good correspondence found above. Even after a thorough investigation of both codes, this discrepancy could not be explained. However, as both codes solve for different variables, it is possible that tiny numerical drifts of the solution in the case of very non-linear cases such as this one are responsible of the differences.

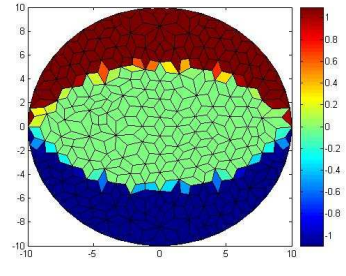

(a) Hybrid FEM-FVM

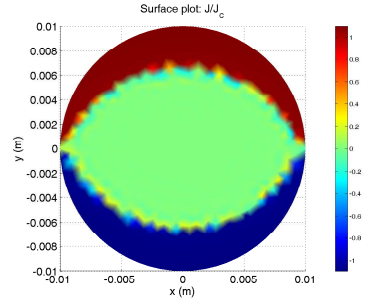

(b) SAM
Fig. 4: Comparison for $n=100$, at $t=T_{0} / 8$

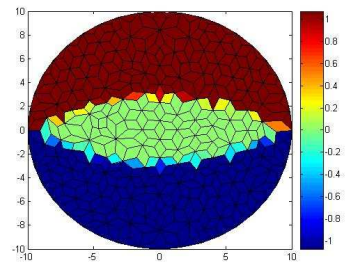

(a) Hybrid FEM-FVM

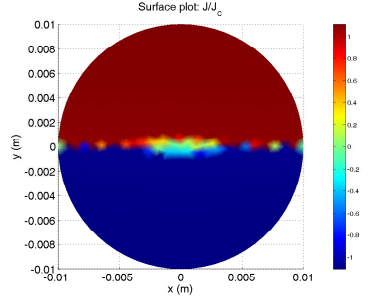

(b) SAM
Fig. 5: Comparison for $n=100$, at $t=T_{0} / 4$

In order to further explore this aspect, we performed a simulation with $n=200$. The result for the HM is presented in Fig. 6 at times $t=T_{0} / 8$ and $t=T_{0} / 4$. The SAM did not converge in this case, and neither did another finite element method code we had access to. In this case, we reached the Bean penetration limit at $t=T_{0} / 4$, which seems reasonable as $n=200$ is a very good approximation of the Bean model. This suggests that our new hybrid method is very robust against strong non-linearities, thanks to the finite volume properties and the change of variable chosen (we solve for $\beta(u)$ instead of $u$ directly).

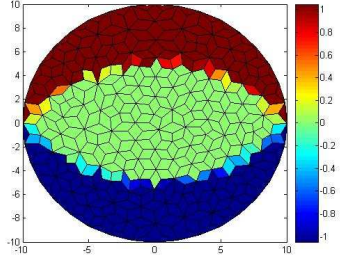

(a) Hybrid FEM-FVM, $\frac{T_{0}}{8}$

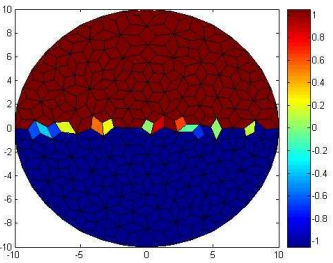

(b) Hybrid FEM-FVM, $\frac{T_{0}}{4}$
Fig. 6: Results of hybrid FEM-FVM, for $n=200$

\section{CONCLUSION}

In this paper, we presented the development of an hybrid FE$\mathrm{FV}$ formulation to solve the non-linear electric field diffusion in 2-D superconducting materials. This method takes advantage of both the FE and FV methods, namely:

$\star$ FEM: Easy to deal with irregular mesh and to process the Laplacian operator

* FVM: Easy to treat the non-linear term $\beta(u)$

Interestingly, the accuracy of the results seems to be independent of mesh refinements. The hybrid method was validated by comparing the results against another validated method in the case of moderate non-linearity (power law exponent of $n=$ 15 ), and proved to be very robust for $n$ values up to 200 . However, for strong non-linearities, it was impossible to obtain identical results between all numerical methods available to us. A full understanding of these discrepancies will require further investigation.

\section{REFERENCES}

[1] M.V. Feigelman, V.B. Geshkenbein, A.I. Larkin and V.M. Vinokur Theory of collective flux creep. Phys rev lett. 63, 2003, 1989

[2] Hernst Helmut Brandt Electric field in superconductors with rectangular cross section. Physhc'l rdvhev B, Vnl 52, Ntm 20, p05422-05456, 1894.

[3] F. Sirois, F. Roy Computation of 2-D Current Distribution in Superconductors of Arbitrary Shapes Using a New Semi-analytical Method. IEEE Transactions on applied superconductivity, Vol 17, Number 3, 2007.

[4] R. Eymard, T. Gallouet, R. Herbin and A. Michel Convergence of a finite volume scheme for non-linear degenerate parabolic equations. Numer Math 92,p41-82, 2002.

[5] P. Charrier Méthode "Cell-centered" pour lapproximation des termes de diffusions sur des maillages non structurés. C.R. Acad Sci. Paris, Serie I 323, p91-94, 1996.

[6] F. Pascal and J.M. Ghidaglia Footbridge between finite volumes and finite elements with applications to CFD. Internat. J. Numer Methods Fluids, 37(8), p951-986, 2001.

[7] R. Eymard, D. Hilhorst, M. Vohralik A combine finite volumenonconforming/Mixed-hybrid finite element scheme for degenerate parabolic problems. Numer Math 105,p73-131, 2006.

[8] R. Dautray, J.L. Lions Analyse mathématique et Calcul numérique. Tome 6. Masson, Paris, 1998.

[9] Jr.W.J. Carr Ac loss and macroscopic theory of superconductors. Second Edition, 2001. 